\title{
Plasmonics and its Applications
}

\author{
Grégory Barbillon $\mathbb{1}$
}

EPF-Ecole d'Ingénieurs, 3 bis rue Lakanal, 92330 Sceaux, France; gregory.barbillon@epf.fr

Received: 1 May 2019; Accepted: 4 May 2019; Published: 8 May 2019

Abstract: Plasmonics is a quickly developing subject that combines fundamental research and applications ranging from areas such as physics to engineering, chemistry, biology, medicine, food sciences, and the environmental sciences. Plasmonics appeared in the 1950s with the discovery of surface plasmon polaritons. Then, plasmonics went through a novel impulsion in mid-1970s when the surface-enhanced Raman scattering was discovered. Nevertheless, it is in this last decade that a very significant explosion of plasmonics and its applications has occurred. Thus, this special issue reports a snapshot of current advances in these various areas of plasmonics and its applications presented in the format of several articles and reviews written by worldwide researchers of this topic.

Keywords: plasmonics; sensing; surface-enhanced Raman scattering; sum-frequency generation; third harmonic generation; surface-enhanced fluorescence; metasurfaces; catalysis; lanthanum hexaboride; solar cell

\section{Introduction}

Plasmonics (or nanoplasmonics) is a young topic of research, which is part of nanophotonics and nano-optics. Plasmonics concerns to the investigation of electron oscillations in metallic nanostructures and nanoparticles (NPs). Surface plasmons have optical properties, which are very interesting. For instance, surface plasmons have the unique capacity to confine light at the nanoscale [1-3]. Moreover, surface plasmons are very sensitive to the surrounding medium and the properties of the materials on which they propagate. In addition to the above, the surface plasmon resonances can be controlled by adjusting the size, shape, periodicity, and materials nature. Indeed, the technological progress allows researchers to produce new plasmonic systems by controlling all the parameters described previously [4-14]. Moreover, theoretical, computational, and numerical simulation tools have been developed in this last decade, allowing for a better understanding of the optical properties of plasmonic systems [1]. Thus, all these optical properties of plasmonic systems can enable a great number of applications, such as biosensors [15-20], optical devices [21-24], and photovoltaic devices [25-28].

\section{Synopsis}

This special issue is composed of five review articles, five research articles, and two communications. The first part of the latter is devoted to the applications of plasmonics to physics and engineering [29-33]. Concerning the applications to physics, such as non-linear optics, Mattox et al. demonstrated the control of plasmonic properties of $\mathrm{LaB}_{6}$ via Eu-doping on a spectral range from near-infrared to infrared [29]. Then, Mattox et al. presented a review on the tuning of the plasmonic resonance of lanthanum hexaboride for a potential application to solar heat absorption [30]. Besides, Ogata et al. investigated the effect of the plasmonic resonance of metallic nanostructures on the optical third harmonic generation (THG) enhancement of nickel nanostructure-covered microcubes [31]. For the application to photovoltaics, Hajiiah et al. presented a simulation study of the efficiency enhancement of peroskite solar cells by using plasmonic nanoparticles [32]. To finish this first part dedicated to physics with the application 
to metasurfaces, Li et al. proposed a novel computational method in order to optimize the coupling of the electric fields of a metasurface consisting of nanorod plasmonic antennas. This novel computational method is based on the coupling of the decomposition into several orders [33].

In the second and last part, the discussed topics are devoted to chemistry and sensing, such as surface-enhanced fluorescence, surface-enhanced Raman scattering (SERS), sum-frequency generation (SFG) spectroscopy, and electrocatalysis by using plasmonics [34-40]. Concerning the surface-enhanced fluorescence, Lu et al. numerically demonstrated a high enhancement effect of the fluorescence signal obtained with a hybrid metal-dielectric nano-aperture antenna consisting of silicon and gold layers [34]. Besides, for the SERS topic, Magno et al. showed excellent analytical enhancement factors of the SERS signal obtained with hybrid $\mathrm{Al} / \mathrm{Si}$ nanopillars for the detection of thiophenol molecules. These hybrid $\mathrm{Al} / \mathrm{Si}$ nanopillars have been realized with a simple and quick fabrication technique [35]. Moreover, Sarychev et al. presented a review on the light concentration by metal-dielectric micro/nano-resonators for efficient SERS sensing. In this review, the recent advances in this topic of metal-dielectric micro/nano-resonators for SERS are exposed [36]. Furthermore, D'Orlando et al. showed the feasibility to carry out and control nanostructures of gold nanoparticles, which can be seen as plasmonic molecules whose optical resonances are tuned by modifying the shape, symmetry, and interparticle distances with an AFM (Atomic Force Microscope) device coupled with an optical spectrometer [37]. To complete the sensing part, Han et al. presented a short review on plasmonic biosensing based on the design of nanovoids in thin films by reviewing resonance modes, materials, and hybrid functions using simultaneously electrical conductivity [38]. In addition, Humbert et al. presented a review on the sum-frequency generation (SFG) spectroscopy of plasmonic nanomaterials. In this review, the authors introduced the fundamentals of SFG spectroscopy. Then, they presented an overview of studies of plasmonic nanomaterials by this SFG spectroscopy over the last five years [39]. To conclude this part, as well as this special issue dedicated to "Plasmonics and its Applications", Subramanian et al. presented a review on the electrocatalysis induced by plasmon with multi-component nanostructures. Indeed, the authors highlight the recent progress obtained in the synthesis of these multi-component nanostructures, especially for the plasmonic electrocatalysis of major fuel-forming and fuel cell reactions [40].

\section{Conclusions}

In making this special issue on plasmonics and its applications, I had the pleasure of obtaining contributions from high-quality authors worldwide, and I thank them for that. To conclude, I hope that this special issue dedicated to plasmonics and its applications will be read with interest by the students or researchers who wish to be involved in this topic or to gain an advanced understanding of it.

Funding: This research received no external funding.

Conflicts of Interest: The authors declare no conflict of interest.

\section{References}

1. Shahbazyan, T.V.; Stockman, M.I. Plasmonics: Theory and Applications; Springer: Dordrecht, The Netherlands, 2013; pp. 1-577.

2. Maier, S.A. Plasmonics: Fundamentals and Applications; Springer: New York, NY, USA, 2007; pp. 3-220.

3. Barbillon, G. Nanoplasmonics-Fundamentals and Applications; InTech: Rijeka, Croatia, 2017; pp. 3-481.

4. Barbillon, G.; Faure, A.-C.; El Kork, N.; Moretti, P.; Roux, S.; Tillement, O.; Ou, M. G.; Descamps, A.; Perriat, P.; Vial, A.; et al. How nanoparticles encapsulating fluorophores allow a double detection of biomolecules by localized surface plasmon resonance and luminescence. Nanotechnology 2008, 19, 035705. [CrossRef] [PubMed]

5. Barbillon, G.; Bijeon, J.-L.; Lérondel, G.; Plain, J.; Royer, P. Detection of chemical molecules with integrated plasmonic glass nanotips. Surf. Sci. 2008, 602, L119-L122. [CrossRef] 
6. Faure, A.-C.; Barbillon, G.; Ou, M.; Ledoux, G.; Tillement, O.; Roux, S.; Fabregue, D.; Descamps, A.; Bijeon, J.-L.; Marquette, C.A.; et al. Core/shell nanoparticles for multiple biological detection with enhanced sensitivity and kinetics. Nanotechnology 2008, 19, 485103. [CrossRef] [PubMed]

7. Bryche, J.-F.; Gillibert, R.; Barbillon, G.; Sarkar, M.; Coutrot, A.-L.; Hamouda, F.; Aassime, A.; Moreau, J.; Lamy de la Chapelle, M.; Bartenlian, B.; et al. Density effect of gold nanodisks on the SERS intensity for a highly sensitive detection of chemical molecules. J. Mater. Sci. 2015, 50, 6601-6607. [CrossRef]

8. Bryche, J.-F.; Gillibert, R.; Barbillon, G.; Gogol, P.; Moreau, J.; Lamy de la Chapelle, M.; Bartenlian, B.; Canva, M. Plasmonic enhancement by a continuous gold underlayer: application to SERS sensing. Plasmonics 2016, 11, 601-608. [CrossRef]

9. Bryche, J.-F.; Tsigara, A.; Bélier, B.; Lamy de la Chapelle, M.; Canva, M.; Bartenlian, B.; Barbillon, G. Surface enhanced Raman scattering improvement of gold triangular nanoprisms by a gold reflective underlayer for chemical sensing. Sens. Actuator B 2016, 228, 31-35. [CrossRef]

10. Masson, J.-F.; Gibson, K.F.; Provencher-Girard, A. Surface-enhanced Raman spectroscopy amplification with film over etched nanospheres. J. Phys. Chem. C 2010, 114, 22406-22412. [CrossRef]

11. Lee, Y.; Lee, J.; Lee, T.K.; Park, J.; Ha, M.; Kwak, S.K.; Ko, H. Particle-on-Film Gap Plasmons on Antireflective ZnO Nanocone Arrays for Molecular-Level Surface-Enhanced Raman Scattering Sensors. ACS Appl. Mater. Interfaces 2015, 7, 26421-26429. [CrossRef] [PubMed]

12. Bryche, J.-F.; Bélier, B.; Bartenlian, B.; Barbillon, G. Low-cost SERS substrates composed of hybrid nanoskittles for a highly sensitive sensing of chemical molecules. Sens. Actuator B 2017, 239, 795-799. [CrossRef]

13. Magno, G.; Bélier, B.; Barbillon, G. Gold thickness impact on the enhancement of SERS detection in low-cost $\mathrm{Au} / \mathrm{Si}$ nanosensors. J. Mater. Sci. 2017, 52, 13650-13656. [CrossRef]

14. Bryche, J.-F.; Barbillon, G.; Bartenlian, B.; Dujardin, G.; Boer-Duchemin, E.; Le Moal, E. k-space optical microscopy of nanoparticle arrays: Opportunities and artifacts. J. Appl. Phys. 2018, 124, 043102. [CrossRef]

15. Barbillon, G.; Noblet, T.; Busson, B.; Tadjeddine, A.; Humbert, C. Localised detection of thiophenol with gold nanotriangles highly structured as honeycombs by nonlinear sum frequency generation spectroscopy. J. Mater. Sci. 2018, 53, 4554-4562. [CrossRef]

16. Dolci, M.; Bryche, J.-F.; Leuvrey, C.; Zafeiratos, S.; Gree, S.; Begin-Colin, S.; Barbillon, G.; Pichon, B.P. Robust clicked assembly based on iron oxide nanoparticles for a new type of SPR biosensor. J. Mater. Chem. C 2018, 6, 9102-9110. [CrossRef]

17. Pichon, B.P.; Barbillon, G.; Marie, P.; Pauly, M.; Begin-Colin, S. Iron oxide magnetic nanoparticles used as probing agents to study the nanostructure of mixed self-assembled monolayers. Nanoscale 2011, 3, 4696-4705. [CrossRef] [PubMed]

18. Barbillon, G. Fabrication and SERS Performances of Metal/Si and Metal/ZnO Nanosensors: A Review. Coatings 2019, 9, 86. [CrossRef]

19. He, Y.; Su, S.; Xu, T.T.; Zhong, Y.L.; Zapien, J.A.; Li, J.; Fan, C.H.; Lee, S.T. Silicon nanowires-based highly-efficient SERS-active platform for ultrasensitive DNA detection. Nano Today 2011, 6, 122-130. [CrossRef]

20. Huang, J.-A.; Zhao, Y.-Q; Zhang, X.-J.; He, L.-F.; Wong, T.-L.; Chui, Y.-S.; Zhang W.-J.; Lee, S.-T. Ordered Ag/Si Nanowires Array: Wide-Range Surface-Enhanced Raman Spectroscopy for Reproducible Biomolecule Detection. Nano Lett. 2013, 13, 5039-5045. [CrossRef]

21. Salamin, Y.; Ma, P.; Baeuerle, B.; Emboras, A.; Fedoryshyn, Y.; Heni, W.; Cheng, B.; Josten, A.; Leuthold, J. $100 \mathrm{GHz}$ Plasmonic Photodetector. ACS Photonics 2018, 5, 3291-3297. [CrossRef]

22. Thomaschewski, M.; Yang, Y.Q.; Bozhevolnyi, S.I. Ultra-compact branchless plasmonic interferometers. Nanoscale 2018, 10, 16178-16183. [CrossRef] [PubMed]

23. Ayata, M.; Fedoryshyn, Y.; Heni, W.; Baeuerle, B.; Josten, A.; Zahner, M.; Koch, U.; Salamin, Y.; Hoessbacher, C.; Haffner, C.; et al. High-speed plasmonic modulator in a single metal layer. Science 2017, 358, 630-632. [CrossRef] [PubMed]

24. Haffner, C.; Heni, W.; Fedoryshyn, Y.; Niegemann, J.; Melikyan, A.; Elder, D.L.; Baeuerle, B.; Salamin, Y.; Josten, A.; Koch, U.; et al. All-plasmonic Mach-Zehnder modulator enabling optical high-speed communication at the microscale. Nat. Photonics 2015, 9, 525-528. [CrossRef]

25. Shao, W.J.; Liang, Z.Q.; Guan, T.F.; Chen, J.M.; Wang, Z.F.; Wu, H.H.; Zheng, J.Z.; Abdulhalim, I.; Jiang, L. One-step integration of a multiple-morphology gold nanoparticle array on a $\mathrm{TiO}_{2}$ film via a facile sonochemical method for highly efficient organic photovoltaics. J. Mater. Chem. A 2018, 6, 8419-8429. [CrossRef] 
26. Vangelidis, I.; Theodosi, A.; Beliatis, M.J.; Gandhi, K.K.; Laskarakis, A.; Patsalas, P.; Logothetidis, S.; Silva, S.R.P.; Lidorikis, E. Plasmonic Organic Photovoltaics: Unraveling Plasmonic Enhancement for Realistic Cell Geometries. ACS Photonics 2018, 5, 1440-1452. [CrossRef]

27. Li, M.Z.; Guler, U.; Li, Y.A.; Rea, A.; Tanyi, E.K.; Kim, Y.; Noginov, M.A.; Song, Y.L.; Boltasseva, A.; Shalaev, V.M.; et al. Plasmonic Biomimetic Nanocomposite with Spontaneous Subwavelength Structuring as Broadband Absorbers. ACS Energy Lett. 2018, 3, 1578-1583. [CrossRef]

28. Chen, X.; Fang, J.; Zhang, X.D.; Zhao, Y.; Gu, M. Optical/Electrical Integrated Design of Core-Shell Aluminum-Based Plasmonic Nanostructures for Record-Breaking Efficiency Enhancements in Photovoltaic Devices. ACS Photonics 2017, 4, 2102-2110. [CrossRef]

29. Mattox, T.M.; Coffman, D.K.; Roh, I.; Sims, C.; Urban, J.J. Moving the Plasmon of LaB 6 from IR to Near-IR via Eu-Doping. Materials 2018, 11, 226. [CrossRef] [PubMed]

30. Mattox, T.M.; Urban, J.J. Tuning the Surface Plasmon Resonance of Lanthanum Hexaboride to Absorb Solar Heat: A Review. Materials 2018, 11, 2473. [CrossRef]

31. Ogata, Y.; Vorobyev, A.; Guo, C. Optical Third Harmonic Generation Using Nickel Nanostructure-Covered Microcube Structures. Materials 2018, 11, 501. [CrossRef]

32. Hajjiah, A.; Kandas, I.; Shehata, N. Efficiency Enhancement of Perovskite Solar Cells with Plasmonic Nanoparticles: A Simulation Study. Materials 2018, 11, 1626. [CrossRef]

33. Li, Y.; Hong, M. Diffractive Efficiency Optimization in Metasurface Design via Electromagnetic Coupling Compensation. Materials 2019, 12, 1005. [CrossRef] [PubMed]

34. Lu, G.; Xu, J.; Wen, T.; Zhang, W.; Zhao, J.; Hu, A.; Barbillon, G.; Gong, Q. Hybrid Metal-Dielectric Nano-Aperture Antenna for Surface Enhanced Fluorescence. Materials 2018, 11, 1435. [CrossRef]

35. Magno, G.; Bélier, B.; Barbillon, G. Al/Si Nanopillars as Very Sensitive SERS Substrates. Materials 2018, 11, 1534. [CrossRef] [PubMed]

36. Sarychev, A.K.; Ivanov, A.; Lagarkov, A.; Barbillon, G. Light Concentration by Metal-Dielectric Micro-Resonators for SERS Sensing. Materials 2019, 12, 103. [CrossRef] [PubMed]

37. D'Orlando, A.; Bayle, M.; Louarn, G.; Humbert, B. AFM-Nano Manipulation of Plasmonic Molecules Used as "Nano-Lens" to Enhance Raman of Individual Nano-Objects. Materials 2019, 12, 1372. [CrossRef] [PubMed]

38. Han, X.; Liu, K.; Sun, C.-S. Plasmonics for Biosensing. Materials 2019, 12, 1411. [CrossRef] [PubMed]

39. Humbert, C.; Noblet, T.; Dalstein, L.; Busson, B.; Barbillon, G. Sum-Frequency Generation Spectroscopy of Plasmonic Nanomaterials: A Review. Materials 2019, 12, 836. [CrossRef]

40. Subramanian, P.; Meziane, D.; Wojcieszak, R.; Dumeignil, F.; Boukherroub, R.; Szunerits, S. Plasmon-Induced Electrocatalysis with Multi-Component Nanostructures. Materials 2019, 12, 43. [CrossRef] [PubMed] 